DESY 10-056

TUM-HEP 758/10

MIT-CTP-4145

\title{
Supersymmetric Leptogenesis with a Light Hidden Sector
}

\author{
Andrea De Simone ${ }^{a}$, Mathias Garny ${ }^{b}$, Alejandro Ibarra ${ }^{b}$, Christoph Weniger $^{c}$ \\ ${ }^{a}$ Center for Theoretical Physics, Massachusetts Institute of Technology, \\ Cambridge, Massachusetts, USA \\ ${ }^{b}$ Physik-Department T30d, Technische Universität München, \\ James-Franck-Straße, 85748 Garching, Germany \\ ${ }^{c}$ Deutsches Elektronen-Synchrotron DESY, Hamburg \\ Notkestraße 85, 22603 Hamburg, Germany
}

\begin{abstract}
Supersymmetric scenarios incorporating thermal leptogenesis as the origin of the observed matter-antimatter asymmetry generically predict abundances of the primordial elements which are in conflict with observations. In this paper we propose a simple way to circumvent this tension and accommodate naturally thermal leptogenesis and primordial nucleosynthesis. We postulate the existence of a light hidden sector, coupled very weakly to the Minimal Supersymmetric Standard Model, which opens up new decay channels for the next-to-lightest supersymmetric particle, thus diluting its abundance during nucleosynthesis. We present a general model-independent analysis of this mechanism as well as two concrete realizations, and describe the relevant cosmological and astrophysical bounds and implications for this dark matter scenario. Possible experimental signatures at colliders and in cosmic-ray observations are also discussed.
\end{abstract}




\section{Introduction}

Extending the Minimal Supersymmetric Standard Model (MSSM) with three heavy right-handed neutrino superfields is one of the best motivated scenarios for physics beyond the Standard Model. The decoupling of the heavy degrees of freedom induces at low energies, after the electroweak symmetry breaking, tiny neutrino masses suppressed by the large right-handed neutrino masses; this is the renown see-saw mechanism [1]. Furthermore, the out of equilibrium decay of the lightest right-handed (s)neutrinos in the early Universe could have generated the observed matter-antimatter asymmetry through the mechanism of leptogenesis [2]. Successful thermal leptogenesis requires, though, a rather large mass scale for the new particles, $M \gtrsim 10^{9} \mathrm{GeV}[3]$, which could destabilize the electroweak scale. Supersymmetry guarantees that the large quadratic quantum corrections to the Higgs mass introduced by the right-handed sneutrinos exactly cancel with the ones introduced by the right-handed neutrinos, thus avoiding the severe hierarchy problem of the non-supersymmetric version of the leptogenesis mechanism.

It is remarkable that this simple scenario can simultaneously address two of the most severe limitations of the Standard Model, namely the existence of non-vanishing neutrino masses and the origin of the observed matter-antimatter asymmetry in our Universe (as well as the above mentioned hierarchy problem). This appealing scenario is nevertheless not exempt of problems. Gravitinos are very efficiently produced in the very hot plasma necessary to generate the observed baryon asymmetry through the mechanism of thermal leptogenesis, the relic density being [4]:

$$
\Omega_{3 / 2}^{\mathrm{th}} h^{2} \simeq 0.27\left(\frac{T_{R}}{10^{9} \mathrm{GeV}}\right)\left(\frac{10 \mathrm{GeV}}{m_{3 / 2}}\right)\left(\frac{m_{\widetilde{g}}}{1 \mathrm{TeV}}\right)^{2},
$$

where $m_{3 / 2}$ is the gravitino mass, $m_{\widetilde{g}}$ is the gluino mass and $T_{R}$ is the reheating temperature of the Universe. If the gravitino is heavier than the lightest neutralino, it decays during or after Big Bang Nucleosynthesis (BBN). Being the gravitinos so abundant at the time of formation of the primordial elements, cf. Eq. (1.1), the large hadronic energy injected into the primeval plasma destroys the successful predictions of the Standard $\mathrm{BBN}$ scenario unless the gravitino mass is larger than $\sim 10^{4} \mathrm{GeV}$ [5, 6].

On the other hand, if the gravitino is lighter than any observable supersymmetric particle, it constitutes a natural candidate for the cold dark matter of the Universe provided it is stable at cosmological scales. Namely, following Eq. (1.1), the dark matter relic density inferred by WMAP for the $\Lambda$ CDM model, $\Omega_{\mathrm{CDM}} h^{2} \simeq 0.11$ [7], can be reproduced for the range of reheating temperatures required by thermal leptogenesis, $T_{R} \gtrsim 10^{9} \mathrm{GeV}$ and for typical gluino masses, $m_{\widetilde{g}} \sim 1 \mathrm{TeV}$, provided the gravitino mass is larger than $\sim 10 \mathrm{GeV}$. 
This attractive scenario is in general in conflict with the observed abundances of primordial elements. If R-parity is conserved, the Lightest Observable Supersymmetric Particle (LOSP) can only decay into Standard Model particles and the gravitino with a decay rate suppressed by the Planck scale, the lifetime being:

$$
\tau_{\text {LOSP }} \simeq 3 \text { days }\left(\frac{m_{3 / 2}}{10 \mathrm{GeV}}\right)^{2}\left(\frac{250 \mathrm{GeV}}{m_{\mathrm{LOSP}}}\right)^{5} .
$$

Therefore, the LOSP is typically present during or after BBN, jeopardizing the successful predictions of the standard nucleosynthesis scenario. This is in fact the case for the most likely candidates for the LOSP: the lightest neutralino and the right-handed stau. More precisely, when the LOSP is the neutralino, the hadrons produced in the neutralino decays typically dissociate the primordial elements [5, 8], yielding abundances in conflict with observations. On the other hand, when the LOSP is a charged particle, $X^{-}$, the formation of the bound state $\left({ }^{4} \mathrm{He} X^{-}\right)$catalyzes the production of ${ }^{6} \mathrm{Li}[9$ ] leading to an abundance of ${ }^{6} \mathrm{Li}$ in stark conflict with observations [10] (for a recent review about BBN constraints see Ref. [1] ).

Different solutions have been proposed to this problem. For instance, in some specific supersymmetric models the LOSP can be a sneutrino [12] or a stop [13], whose late decays do not substantially affect the predictions of BBN. For neutralino or stau LOSP a possible solution consists in introducing a small amount of $R$-parity violation, so that the LOSP decays into two Standard Model particles before the onset of BBN, thus avoiding the BBN constraints altogether [14]. Maintaining the requirement of R-parity conservation, other solutions are to assume a large left-right mixing of the stau mass eigenstates [15], a LOSP mass that is nearly degenerate with the gravitino mass [16], or to assume some amount of entropy production after LOSP decoupling, which dilutes the LOSP abundance [17].

In this paper we would like to propose a scenario which yields a thermal history of the Universe consistent with supersymmetric dark matter and with baryogenesis through thermal leptogenesis, without altering the successful predictions of the Standard BBN scenario 1 We will assume the existence of a hidden sector fermion, $X$, lighter than the LOSP. Thus, new decay channels are possible for the LOSP, for instance, when the LOSP is the lightest stau or the lightest neutralino,

$$
\begin{aligned}
\tilde{\tau}_{1} & \rightarrow \tau X, \\
\chi_{1}^{0} & \rightarrow\left(Z^{0}, \gamma, h^{0}, f \bar{f}\right) X .
\end{aligned}
$$

If these decays are fast enough, the density of LOSPs at the time of nucleosynthesis can be significantly reduced and thus the successful predictions of the standard BBN

\footnotetext{
${ }^{1}$ See also Ref. 18 for a related proposal with light axinos, however without supersymmetric dark matter.
} 
scenario will not be jeopardized. If the gravitino is the LSP, the hidden sector fermion will eventually decay into the gravitino and other particles, hidden or observable. The late decays into gravitinos and hidden sector particles, if kinematically possible, may disrupt the abundances of hidden sector primordial nuclei, but not the abundances of the observed primordial nuclei. On the other hand, the decays into gravitinos and observable particles occur at a rate much larger than the age of the Universe, as we will show, thus not affecting primordial nucleosynthesis. Lastly, if the gravitino is not the LSP it will decay into hidden sector particles. Again, these decays may disrupt the primordial abundances of hidden sector nuclei, but will not have any impact on the standard BBN predictions. This mechanism is sketched in Fig.1, for the case where the gravitino is the LSP (left panel) and for the case where the gravitino can decay into hidden sector particles (right panel).

The couplings of the hidden sector fermion to the MSSM particles are subject to a series of constraints which will be discussed in Section 2. In Section 3 we present two concrete models where the mechanism sketched above can be implemented. In Section 4 we will comment on possible signatures of this scenario at colliders or at cosmic-ray observations. Lastly, in Section 5, we will present our conclusions.

\section{Hidden sector couplings to the MSSM}

We will consider in this paper a scenario where the MSSM particle content is extended with a light superfield (chiral or vector), which is a singlet under the Standard Model gauge group. We will further assume that the fermionic component of this superfield couples to the LOSP and its Standard Model counterpart via a tiny Yukawa coupling, hence we will refer to this fermion as "hidden fermion". In this section we will carefully discuss the implications for leptogenesis of the existence of such hidden fermion, as well as the constraints on this scenario from BBN and from structure formation. Let us first discuss the case of stau LOSP and later on the case of neutralino LOSP.

\section{$2.1 \quad$ Stau LOSP}

The interaction Lagrangian between the hidden fermion and the lightest stau, $\widetilde{\tau}_{1}$, is given by the renormalizable term

$$
-\mathcal{L}=\lambda_{\widetilde{\tau}_{1}} \bar{X} \tau \widetilde{\tau}_{1}+\text { h.c. }
$$


Then the stau can decay either $\widetilde{\tau}_{1} \rightarrow \psi_{3 / 2} \tau$ or $\widetilde{\tau}_{1} \rightarrow X \tau$ with decay rates:

$$
\begin{gathered}
\Gamma_{\widetilde{\tau}_{1} \rightarrow \psi_{3 / 2} \tau} \simeq \frac{1}{48 \pi} \frac{m_{\widetilde{\tau}_{1}}^{5}}{m_{3 / 2}^{2} m_{P}^{2}}\left(1-\frac{m_{3 / 2}^{2}}{m_{\widetilde{\tau}_{1}}^{2}}\right)^{4}, \\
\Gamma_{\widetilde{\tau}_{1} \rightarrow X \tau} \simeq \frac{\left|\lambda_{\widetilde{\tau}_{1}}\right|^{2} m_{\widetilde{\tau}_{1}}}{8 \pi}\left(1-\frac{m_{X}^{2}}{m_{\widetilde{\tau}_{1}}^{2}}\right)^{2},
\end{gathered}
$$

where the tau mass has been neglected. If the coupling $\lambda_{\widetilde{\tau}_{1}}$ is large enough, the stau will decay before it can form bound states with ${ }^{4} \mathrm{He}$, thus preventing the catalytic production of ${ }^{6} \mathrm{Li}$. More concretely, the requirement that the lightest stau decays before $\simeq 2 \times 10^{3} \mathrm{~s}$ [9, 10] into $X \tau$, implies the following lower bound on the coupling $X-\tau-\widetilde{\tau}_{1}$ :

$$
\left|\lambda_{\widetilde{\tau}_{1}}\right| \gtrsim 5 \times 10^{-15}\left(\frac{250 \mathrm{GeV}}{m_{\widetilde{\tau}_{1}}}\right)^{1 / 2}\left(1-\frac{m_{X}^{2}}{m_{\widetilde{\tau}_{1}}^{2}}\right)^{-1} .
$$

If this condition is satisfied, the lightest stau will by itself not play any role during BBN.

A necessary requirement for the viability of this mechanism is that cosmological constraints, namely from thermal overproduction of the hidden fermion $X$ and from structure formation, are satisfied. Different scenarios can arise depending on whether the LSP is the gravitino or the hidden fermion, and on whether the NLSP is stable on cosmological time-scales or not. Below we will discuss each case separately.

\subsubsection{Cosmologically stable gravitino and hidden fermion}

If the hidden fermion is the lightest particle in the hidden sector, its decay channels into a gravitino and other hidden sector particles are kinematically forbidden (note that in this case the hidden fermion still can decay into a gravitino and Standard Model particles, e.g. $X \rightarrow \psi_{3 / 2} \tau^{+} \tau^{-}, X \rightarrow \psi_{3 / 2} \gamma$, with lifetimes which can be larger than the age of the Universe, as will be discussed in Section 4). If this is the case, two particle species contribute to the dark matter, namely gravitinos and hidden fermions, each of them having a thermal component and a non-thermal component:

$$
\Omega_{\mathrm{dm}}=\Omega_{3 / 2}^{\mathrm{th}}+\Omega_{3 / 2}^{\widetilde{\tau}_{1}}+\Omega_{X}^{\mathrm{th}}+\Omega_{X}^{\widetilde{\tau}_{1}}
$$

Here, $\Omega_{3 / 2}^{\widetilde{\tau}_{1}}$ and $\Omega_{X}^{\widetilde{\tau}_{1}}$ are the non-thermal contributions to the gravitino and hidden fermion relic density, respectively, which are given by

$$
\begin{aligned}
\Omega_{3 / 2}^{\widetilde{\tau}_{1}} & =\frac{m_{3 / 2}}{m_{\widetilde{\tau}_{1}}} \operatorname{BR}\left(\widetilde{\tau}_{1} \rightarrow \psi_{3 / 2} \tau\right) \Omega_{\widetilde{\tau}_{1}}^{\text {th }}, \\
\Omega_{X}^{\widetilde{\tau}_{1}} & =\frac{m_{X}}{m_{\widetilde{\tau}_{1}}} \operatorname{BR}\left(\widetilde{\tau}_{1} \rightarrow X \tau\right) \Omega_{\widetilde{\tau}_{1}}^{\text {th }},
\end{aligned}
$$



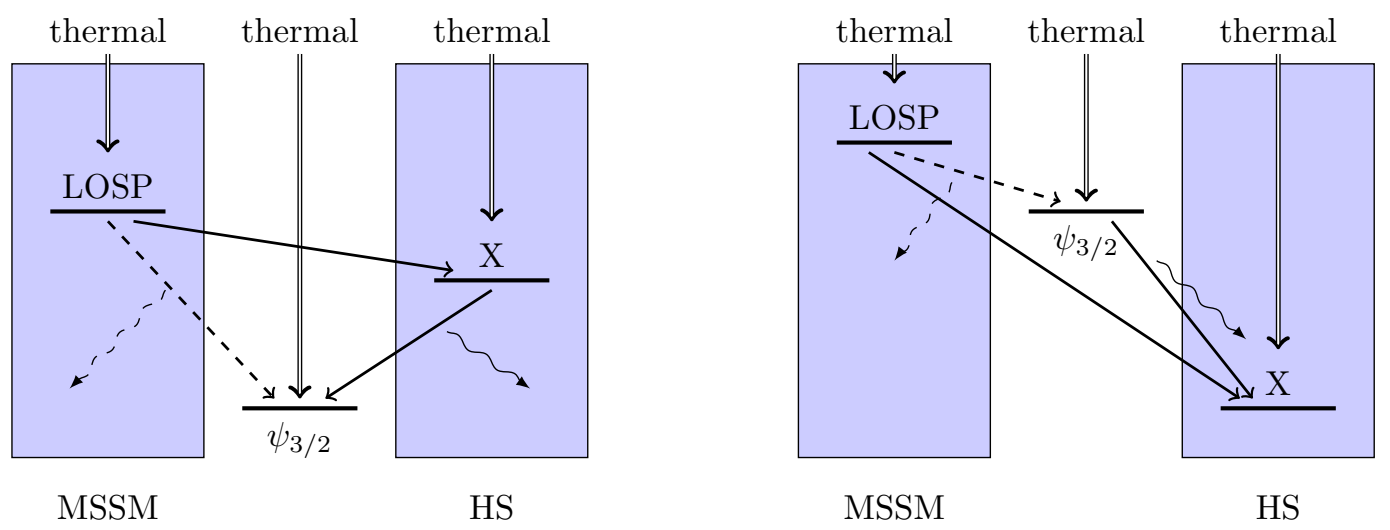

Figure 1: Sketch of our proposed mechanism, for the cases where the LSP is the gravitino (left panel) or a hidden fermion (right panel). The Lightest Observable Supersymmetric Particle (LOSP), the gravitino and the hidden fermion can be produced thermally or non-thermally through the decays of heavier particles; slow decays are indicated with dashed arrows and fast decays with solid arrows, whereas the other decay products are indicated with wiggled lines. If thermal leptogenesis is the correct mechanism to explain the observed matter-antimatter asymmetry, the LOSP decay into the gravitino occurs during or after the time of primordial nucleosynthesis, altering the predictions of the Standard BBN scenario. If this is the only decay channel of the LOSP the impact is usually dramatic, yielding abundances in conflict with observations. However, if the LOSP coupling to the hidden fermion is much larger than the coupling to the gravitino, this decay can occur before the onset of the nucleosynthesis reactions, thus avoiding altogether any possible effect of the LOSP on nucleosynthesis. Eventually the hidden fermion will decay into the gravitino and other hidden sector particles (left panel) or vice versa (right panel). Nevertheless, these decays do not alter the abundances of primordial elements in our observable sector.

where $\Omega_{\widetilde{\tau}_{1}}^{\text {th }}$ is the stau thermal abundance

$$
\Omega_{\widetilde{\tau}_{1}}^{\mathrm{th}} h^{2} \simeq 2 \times 10^{-3}\left(\frac{m_{\widetilde{\tau}_{1}}}{100 \mathrm{GeV}}\right)^{2}
$$

corresponding to a yield $Y_{\widetilde{\tau}_{1}} \simeq 7 \times 10^{-14}\left(m_{\widetilde{\tau}_{1}} / 100 \mathrm{GeV}\right)[19]$. Furthermore, $\Omega_{3 / 2}^{\text {th }}$ is the contribution to the total dark matter density from thermally produced gravitinos, Eq. (1.1). If the hidden fermion couples to the observable sector through a renormalizable coupling, the thermal production proceeds dominantly via the decay of thermally produced staus at temperatures $T \sim m_{\widetilde{\tau}_{1}}$. The corresponding hidden fermion relic abundance $\Omega_{X}^{\text {th }}$ is 
given by 20 ]

$$
\begin{aligned}
\Omega_{X}^{\text {th }} h^{2} & \simeq \frac{1.09 \times 10^{27}}{g_{*}^{3 / 2}} \frac{2 m_{X} \Gamma_{\widetilde{\tau}_{1} \rightarrow \tau X}}{m_{\widetilde{\tau}_{1}}^{2}}(1+\delta) \\
& \simeq 8.6 \times 10^{22}(1+\delta)\left|\lambda_{\widetilde{\tau}_{1}}\right|^{2}\left(\frac{100}{g_{*}}\right)^{3 / 2}\left(\frac{m_{X}}{m_{\widetilde{\tau}_{1}}}\right)\left(1-\frac{m_{X}^{2}}{m_{\widetilde{\tau}_{1}}^{2}}\right)^{2}
\end{aligned}
$$

Here, $g_{*} \approx 100$ denotes the effective number of degrees of freedom at temperature $T \sim$ $m_{\widetilde{\tau}_{1}}$, and $\delta$ parameterizes the potential enhancement of the hidden fermion abundance due to possible additional couplings of the hidden fermion to other MSSM particles, cf. Eq. (3.9) below. Here we set for simplicity $\delta=0$.

In order to sufficiently reduce the number density of staus at the time of BBN it is necessary that $\operatorname{BR}\left(\widetilde{\tau}_{1} \rightarrow \tau X\right) \simeq 1$, and therefore, $\Omega_{3 / 2}^{\widetilde{\tau}_{1}} \simeq 0$. Requiring that the total dark matter density does not exceed the measured value by WMAP implies then

$$
\Omega_{3 / 2}^{\mathrm{th}}+\Omega_{X}^{\mathrm{th}}+\frac{m_{X}}{m_{\widetilde{\tau}_{1}}} \Omega_{\widetilde{\tau}_{1}}^{\text {th }} \lesssim 0.11 h^{-2}
$$

As long as the thermal production of $X$ and staus is small (which also implies a small coupling $\lambda_{\widetilde{\tau}_{1}}$ ) this bound reduces to the standard overproduction constraint on thermally produced gravitinos, $\Omega_{3 / 2}^{\text {th }} h^{2} \lesssim 0.11$, which by its own allows high reheating temperatures $T_{R} \gtrsim 10^{9} \mathrm{GeV}$, as required by leptogenesis, provided the gravitino mass is large enough, see Eq. (1.1). On the other hand, in the regime where the production of $X$ is sizeable, Eqs. (2.9) and (2.10) combine to a bound on the coupling

$$
\left|\lambda_{\widetilde{\tau}_{1}}\right| \lesssim 10^{-12}\left(\frac{m_{\widetilde{\tau}_{1}}}{m_{X}}\right)^{1 / 2}\left(1-\frac{m_{X}^{2}}{m_{\widetilde{\tau}_{1}}^{2}}\right)^{-1}
$$

which is independent of the gravitino mass.

The results are illustrated by the red lines in Fig. 2 for the case of gravitino LSPs and in Fig. 3 for the case of hidden fermion LSPs. The lines show, for different reheating temperatures and different masses of the hidden fermion, the value of the coupling $\lambda_{\widetilde{\tau}_{1}}$ as a function of the gravitino mass from the requirement that the total dark matter density is equal to the value inferred by the WMAP collaboration. In these plots, it was assumed for definiteness $m_{\widetilde{\tau}_{1}}=250 \mathrm{GeV}$. On the other hand, the shaded regions correspond to choices of parameters where the stau decays with a lifetime longer than $2 \times 10^{3} \mathrm{~s}$, thus leading to ${ }^{6} \mathrm{Li}$ overproduction. As apparent from the plots, in both cases of hidden fermion LSP and gravitino LSP, there is a fairly wide region of the parameter space where the reheating temperature can be large enough to allow thermal leptogenesis while preserving the successful predictions of the standard BBN scenario. 


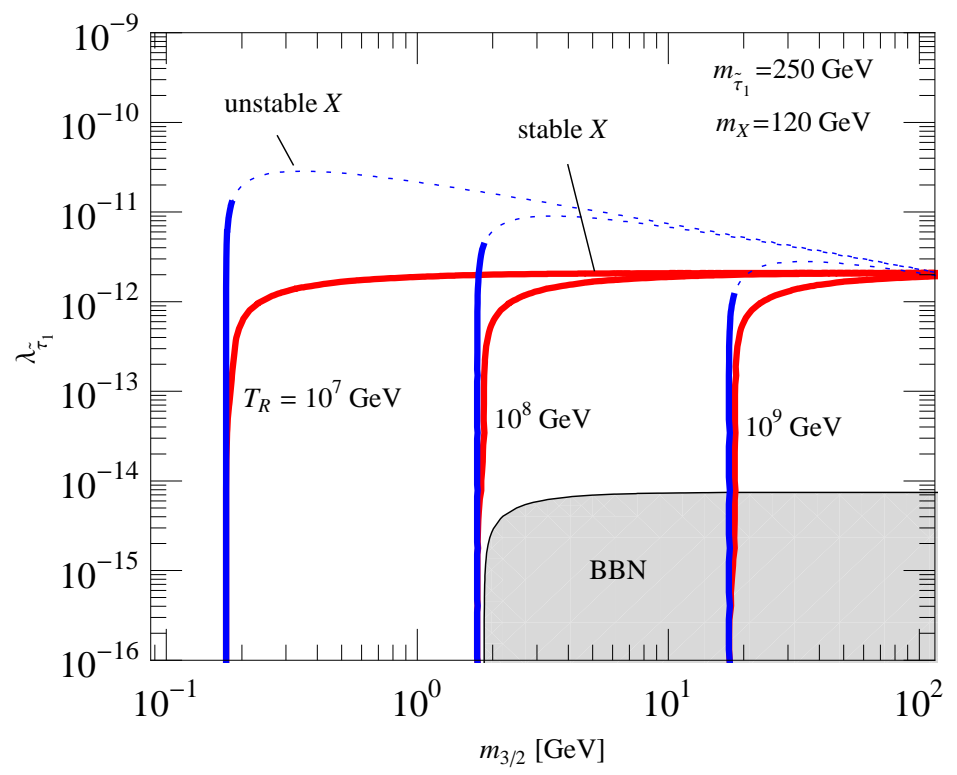

Figure 2: Summary of constraints on stau LOSP scenario with gravitino LSP, as derived from Eq. (2.1), as function of the stau-tau-hidden fermion Yukawa-coupling $\lambda_{\widetilde{\tau}_{1}}$ and gravitino mass $m_{3 / 2}$. The masses of $X$ and $\widetilde{\tau}_{1}$ are fixed as indicated. The red and blue lines show the values of $m_{3 / 2}$ and $\lambda_{\widetilde{\tau}_{1}}$ that yield the correct total relic abundance for different reheating temperatures $T_{R}=10^{7}-10^{9} \mathrm{GeV}$, assuming that $X$ is stable or unstable, respectively (the gluino mass has been set to $m_{\tilde{g}}=800 \mathrm{GeV}$ ). Furthermore, the dashed part of the blue lines is excluded by constraints on mixed warm/cold dark matter as discussed in the text, and the gray region is excluded by ${ }^{6} \mathrm{Li}$ overproduction during $\mathrm{BBN}$. It is clear that for vanishing coupling to the hidden sector, reheating temperatures around $10^{9} \mathrm{GeV}$, as required by thermal leptogenesis, are in conflict with BBN whereas an allowed window opens up for non-zero $\lambda_{\widetilde{\tau}_{1}}$.

\subsubsection{Unstable hidden fermion}

If the gravitino is the LSP, and if kinematically allowed, the hidden fermion $X$ decays into gravitinos and hidden sector particles well before matter-radiation equality. Note that the particles produced in the decay interact very weakly with the particles in the observable sector, therefore the late decays of the hidden fermion do not modify the abundances of primordial elements in the observable sector. In this scenario, the dark matter consists of thermally produced gravitinos, with a relic density given by Eq. (1.1), and non-thermally produced gravitinos, coming from the late decay of hidden fermions 
$X$ and staus $\widetilde{\tau}_{1}$. The dark matter abundance is then given by

$$
\Omega_{\mathrm{dm}}=\Omega_{3 / 2}^{\mathrm{th}}+\underbrace{\Omega_{3 / 2}^{\widetilde{\tau}_{1}}+\frac{m_{3 / 2}}{m_{X}}\left(\Omega_{X}^{\mathrm{th}}+\Omega_{X}^{\widetilde{\tau}_{1}}\right)}_{=\Omega_{\mathrm{WDM}}},
$$

where we assumed for simplicity that the hidden-sector particles produced in the decay of $X$ are massless or very light and hence contribute negligibly to the relic abundance 2 The component coming from the late decay of $X$, as well as the small fraction of gravitinos produced directly in $\widetilde{\tau}_{1}$ decays, will typically act as warm dark matter (WDM), with freestreaming lengths $\lambda_{\text {FS }} \gtrsim 5$ Mpc ( $c f$. Fig. 4 in Ref. [21]) 3 Here, the free-streaming length of a particle is defined as the distance the particle has traveled between its production and the onset of structure formation. It is given by

$$
\lambda_{\mathrm{FS}}=\int_{3000}^{z_{p}} d z \frac{v(z)}{H(z)},
$$

where $z_{p}$ denotes the red-shift at which the particle is produced and is a function of the lifetime of the parent particle, $z \sim 3000$ is the redshift at matter-radiation equality, $v(z)$ is the particle's velocity and $H(z)$ denotes the Hubble parameter as function of redshift, see e.g. Ref. [22].

Observations of the power spectrum of high-redshift Hydrogen clouds via the Lyman$\alpha$ forest [23] imply the upper bound $\lambda_{\mathrm{FS}} \lesssim 0.5 \mathrm{Mpc}$ [24], when all dark matter components have comparable free-streaming lengths. This bound relaxes if a large fraction of the dark matter is cold and just a small fraction of it is warm. Bounds on the fraction $f$ of the dark matter density that is allowed to be warm with a free-streaming length above $0.5 \mathrm{Mpc}$, were discussed in Ref. [25] in the context of sterile neutrinos. There, using Lyman- $\alpha$ data [26] and WMAP5 results, $2 \sigma$-bounds around $f \lesssim 0.05$ were found for a warm component with free-streaming lengths around $\mathcal{O}(10 \mathrm{Mpc})$, corresponding to $\mathcal{O}(1 \mathrm{~km} / \mathrm{s})$ thermal velocities.

For definiteness we will take $f=0.05$ throughout this paper, which implies, following Eq. (2.12), the requirement of a small thermal abundance of $\widetilde{\tau}_{1}$ and $X$. Allowing a fraction $f$ of dark matter to be warm, and provided that $\Omega_{3 / 2}^{\widetilde{\tau}_{1}} \simeq 0$, gives then

$$
\frac{m_{3 / 2}}{m_{X}}\left(\Omega_{X}^{\text {th }}+\Omega_{X}^{\widetilde{\tau}_{1}}\right) \lesssim f 0.11 h^{-2}
$$

\footnotetext{
${ }^{2}$ Otherwise they would contribute to the warm dark matter component and they could cause dangerous late decays into Standard Model particles.

${ }^{3}$ Note that this could also be relevant for $\Omega_{X}^{\widetilde{\tau}_{1}}$ in the above case where gravitinos and hidden fermions are stable, since for small hidden gaugino masses the free-streaming length becomes large. However, in the example shown in Fig. 2, the red lines feature always free-streaming lengths below $\lambda_{\mathrm{FS}} \ll 0.5 \mathrm{Mpc}$ in the region where $\lambda_{\widetilde{\tau}_{1}} \gtrsim 10^{-14}$. Furthermore, since the stau yield is small, the impact of this component on mixed warm/cold dark matter bounds is negligible even if the free-streaming lengths are large.
} 


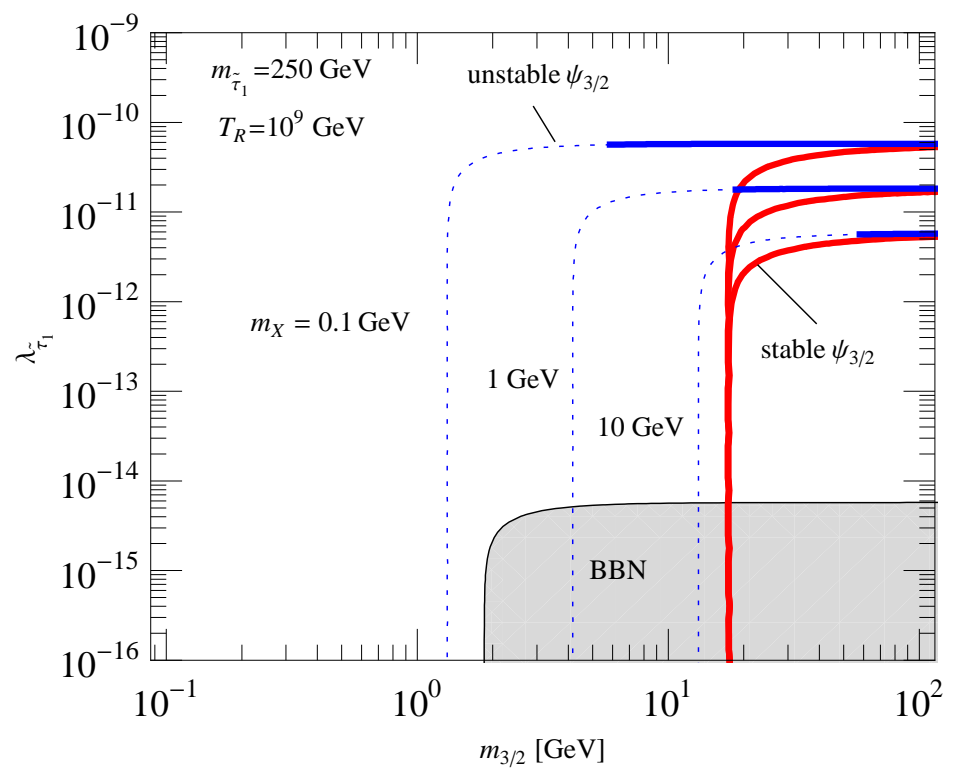

Figure 3: Summary of constraints on stau LOSP scenario with hidden fermion LSP and gravitino NLSP, similar to Fig. 2. Here, the mass of the hidden fermion $X$ is assumed to lie in the range $m_{X}=0.1-10 \mathrm{GeV}$, whereas the reheating temperature and the $\widetilde{\tau}_{1}$ mass are fixed as indicated. The red and blue lines show the constraints for the case of a stable or unstable gravitino, respectively. Dashed parts of the lines are again excluded by constraints on mixed warm/cold dark matter.

In addition to this constraint, the viability of the present scenario requires that both, the thermal gravitino abundance and the thermal hidden fermion abundance, do not exceed the total dark matter density, namely $\Omega_{3 / 2}^{\text {th }}+\left(m_{3 / 2} / m_{X}\right) \Omega_{X}^{\text {th }} \lesssim 0.11 h^{-2}$, cf. Eq. (2.9).

The impact of all these constraints on the parameter space of the scenario is shown in Fig. 2 as blue lines. The dashed part of the lines is excluded from the constraints on mixed warm/cold dark matter, Eq. (2.14). Compared to the scenario where the hidden fermion is stable, now larger values of the coupling $\lambda_{\widetilde{\tau}_{1}}$ are allowed, since the hidden fermion does not directly contribute to the total dark matter abundance any more.

\subsubsection{Unstable gravitino}

In some scenarios the hidden fermion could be the LSP. If this is the case, the gravitino can decay into it, if kinematically allowed, yielding a scenario which is qualitatively different to the one studied in the previous subsection. The total dark matter abundance 
is given in this case by:

$$
\Omega_{\mathrm{dm}}=\Omega_{X}^{\mathrm{th}}+\Omega_{X}^{\widetilde{\tau}_{1}}+\underbrace{\frac{m_{X}}{m_{3 / 2}}\left(\Omega_{3 / 2}^{\mathrm{th}}+\Omega_{3 / 2}^{\widetilde{\tau}_{1}}\right)}_{=\Omega_{\mathrm{WDM}}},
$$

where we assumed that hidden-sector by-products of the gravitino decaying into $X$ are massless 4 In this case, the thermal production of gravitinos itself produces ultimately WDM, yielding the constraint

$$
\Omega_{3 / 2}^{\mathrm{th}} h^{2} \lesssim \frac{m_{3 / 2}}{m_{X}} f 0.11
$$

where we again assumed that $\Omega_{3 / 2}^{\widetilde{\tau}_{1}} \simeq 0$. It is apparent from this equation that in scenarios with unstable gravitinos and high reheating temperatures, small masses $m_{X} \lesssim f m_{3 / 2}$ are favored, to avoid strengthening the bounds on thermal gravitino production. Furthermore, the thermal or non-thermal production of $X$ becomes essential, since it must yield the dominant part of the dark matter abundance according to $\Omega_{X}^{\text {th }}+\Omega_{X}^{\widetilde{\tau}_{1}} \simeq h^{-2} 0.11$, which implies a coupling to the hidden fermion like $\lambda_{\widetilde{\tau}_{1}} \approx 10^{-12}\left(m_{\widetilde{\tau}_{1}} / m_{X}\right)^{1 / 2}$, as long as $\Omega_{X}^{\widetilde{\tau}_{1}}$ is negligible.

This situation is illustrated by the blue lines in Fig. 3, for fixed reheating temperature $T_{R}=10^{9} \mathrm{GeV}$ and for different masses of the hidden fermion $m_{X}$. As apparent from this plot, for being in agreement with the bounds on mixed warm/cold dark matter the gravitino mass has to be considerably higher than $m_{X}$ in each of the shown cases, e.g. for $m_{X}=1 \mathrm{GeV}$ the gravitino mass should exceed $20 \mathrm{GeV}$. Typical couplings where the mechanism works lie in the range $\lambda_{\widetilde{\tau}_{1}} \sim 10^{-12}-10^{-10}$.

\subsection{Neutralino LOSP}

We will now briefly discuss the case of neutralino LOSP. For definiteness, we consider an effective interaction Lagrangian between the hidden fermion $X$ and the lightest neutralino $\chi_{1}^{0}$ given by

$$
-\mathcal{L}=g_{h} \bar{X} \chi_{1}^{0} h^{0}+g_{Z} \bar{X} \gamma_{\mu} \chi_{1}^{0} Z^{\mu}+g_{\gamma} \bar{X} \gamma_{\mu} \chi_{1}^{0} A^{\mu}+\text { h.c. }
$$

The couplings $g_{i}$ include gauge couplings, weak mixing angles etc. The neutralino can decay either $\chi_{1}^{0} \rightarrow\left(Z^{0}, \gamma, h^{0}\right) \psi_{3 / 2}$ or $\chi_{1}^{0} \rightarrow\left(Z^{0}, \gamma, h^{0}\right) X$.

The electromagnetic and hadronic energy $\epsilon_{v i s}=\left(m_{\chi_{1}^{0}}^{2}-m_{\psi_{3 / 2} / X}^{2}+m_{Z / h}^{2}\right) /\left(2 m_{\chi_{1}^{0}}\right)$ released during these decays can induce photo- and hadrodissociation of ${ }^{4} \mathrm{He}$ during

\footnotetext{
${ }^{4}$ Note that here again the component $\Omega_{X}^{\widetilde{\tau}_{1}}$ has a large free-streaming length $\lambda_{F S} \gtrsim 1 \mathrm{Mpc}$ in some cases, depending on the coupling $\lambda_{\widetilde{\tau}_{1}}$ and the mass $m_{\widetilde{\tau}_{1}}$. However, due to the small stau (and later the neutralino) yield, the impact of this component is always negligible in our plots.
} 
$\mathrm{BBN}$, and can lead to inter-conversions of protons and neutrons [5, 8]. Without hidden fermion $X$ (corresponding to the limits $g_{i} \rightarrow 0$ ), this leads to stringent constraints on the gravitino mass and consequently also on the reheating temperature [27]. However, if the couplings $g_{i}$ are large enough, the neutralino decays into hidden fermions already before $\mathrm{BBN}$, allowing a high reheating temperature $T_{R} \sim 10^{9} \mathrm{GeV}$ as required by leptogenesis.

As an example we will consider the decay $\chi_{1}^{0} \rightarrow Z^{0} X$, where the decay width is given by

$$
\begin{aligned}
\Gamma_{\chi_{1}^{0} \rightarrow Z^{0} X}= & \frac{g_{Z}^{2}}{32 \pi} m_{\chi_{1}^{0}}\left[1+\frac{m_{X}^{2}}{m_{\chi_{1}^{0}}^{2}}-2 \frac{m_{Z}^{2}}{m_{\chi_{1}^{0}}^{2}}+\frac{m_{\chi_{1}^{0}}^{2}}{m_{Z}^{2}}\left(1-\frac{m_{X}^{2}}{m_{\chi_{1}^{0}}^{2}}\right)^{2}-6 \frac{m_{X}}{m_{\chi_{1}^{0}}}\right] \\
& \times \sqrt{\left[1-\frac{\left(m_{X}+m_{Z}\right)^{2}}{m_{\chi_{1}^{0}}^{2}}\right]\left[1-\frac{\left(m_{X}-m_{Z}\right)^{2}}{m_{\chi_{1}^{0}}^{2}}\right]},
\end{aligned}
$$

assuming that only $g_{Z} \neq 0$. The $Z^{0}$ boson then decays into hadrons with branching ratio $B_{h} \sim 0.7$.

For a reference neutralino yield of $\epsilon_{v i s} Y_{\chi_{1}^{0}} \sim 100 \mathrm{GeV} \times 10^{-12}$ [28], the most stringent constraints come from overproduction of ${ }^{4} \mathrm{He}$ due to interconversion processes, as well as D production by hadrodissociation, leading to an upper bound on the neutralino lifetime in the range $\tau_{\chi_{1}^{0}}<\tau_{\max } \sim 1-100 \mathrm{~s}$ [5, 8]. In the parameter region consistent with thermal leptogenesis, the decay into gravitinos is negligible, and $\operatorname{BR}\left(\chi_{1}^{0} \rightarrow Z^{0} X\right) \simeq 1$. This implies a lower bound on the coupling, which reads in the limit $m_{\chi_{1}^{0}} \gg m_{Z}, m_{X}$ :

$$
\left|g_{Z}\right| \gtrsim 3 \times 10^{-14}\left(\frac{m_{\chi_{1}^{0}}}{200 \mathrm{GeV}}\right)^{-3 / 2}\left(\frac{\tau_{\max }}{100 \mathrm{~s}}\right)^{-1 / 2}
$$

The constraints from overclosure and from free-streaming are very similar to the case of stau LOSP discussed before, and we do not repeat them here. The corresponding constraints for $Y_{\chi_{1}^{0}}=10^{-12}$ are summarized in Fig. 4. In this figure, we also took the decay modes $\chi_{1}^{0} \rightarrow\left(Z^{0}, \gamma\right) \psi_{3 / 2}$ into account using the rates given in [27], assuming a bino-like neutralino 5 Note that for both cases, gravitino and hidden fermion LSPs, one can find parameters compatible with $T_{R} \sim 10^{9} \mathrm{GeV}$, and typical mixing parameters lie in the range $g_{Z} \sim 10^{-13}-10^{-11}$.

The bounds shown in Fig. 4 are relying on the relatively small adopted neutralino yield, and they can change qualitatively when the yield is much larger. For example, in the case where the hidden fermion is the NLSP and unstable (blue lines in the left panel of Fig. (4), the warm dark matter component produced by the decay chain $\chi_{1}^{0} \rightarrow X \rightarrow \psi_{3 / 2}$

\footnotetext{
${ }^{5}$ The BBN bounds were obtained by interpolating between the bounds for the different hadronic branching ratios given in 8 .
} 

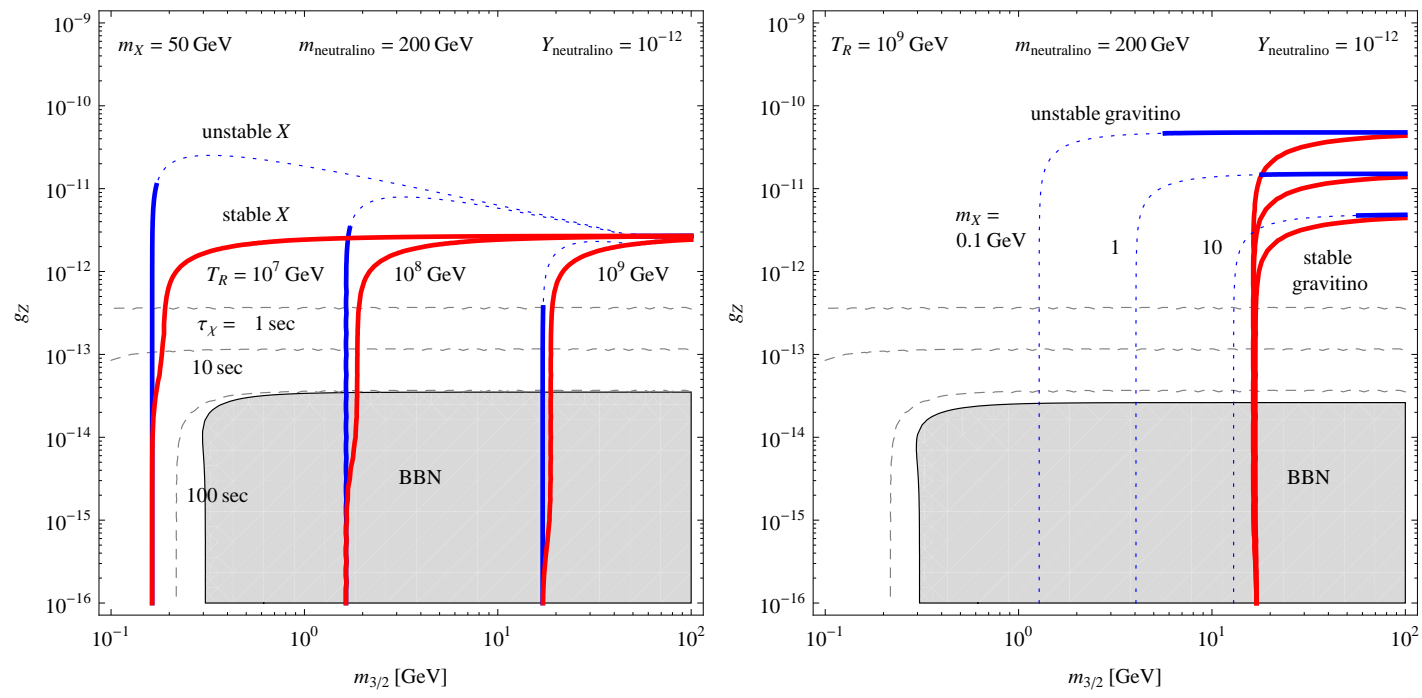

Figure 4: Summary of constraints on neutralino LOSP scenario with gravitino LSP (left panel, as in Fig. 2), and hidden fermion LSP (right panel, as in Fig. 3), as function of the $\chi_{1}^{0} X Z^{0}$ coupling $g_{Z}$ and gravitino mass $m_{3 / 2}$, and for $Y_{\chi_{1}^{0}}=10^{-12}$. The constraints from BBN obtained in Ref. [8] (shaded region) exclude neutralino life-times $\tau_{\chi_{1}^{0}} \gtrsim 100 \mathrm{~s}$. However, Ref. [5] presents somewhat stronger bounds, of the order 1-10s (grey dashed lines).

implies an upper limit on the neutralino yield,

$$
Y_{\chi_{1}^{0}} \lesssim 10^{-12} \frac{20 \mathrm{GeV}}{m_{3 / 2}} \frac{f}{0.05}
$$

which follows from the bounds on mixed warm/cold dark matter. On the other hand, in the cases where the gravitino is the NLSP, one can find viable scenarios even for much higher values of the neutralino yield, which however requires that the hidden fermion is very light.

\section{Examples}

After having discussed the proposed mechanism as well as astrophysical and cosmological bounds in the last section in general, we will now present two concrete scenarios with hidden fermions from a vector and from a chiral supermultiplet, respectively. 


\subsection{Hidden gauginos of an unbroken $U(1)_{X}$}

Let us consider the case where the hidden fermion arises from the gaugino component of a vector superfield of an unbroken hidden $U(1)_{X}$ symmetry, which mixes with the $U(1)_{Y}$ of hypercharge via a small kinetic mixing $\chi \ll 1$ (for details see also Ref. [21]). This scenario is an example for the case where the superpartner of the hidden fermion $X$, here the $U(1)_{X}$ vector boson, remains exactly massless. For simplicity, we will assume that all matter charged under $U(1)_{X}$ is vector-like and heavy enough to be cosmologically irrelevant.

When SUSY is exact, the canonical normalization of the kinetic terms of the vector superfields produces an unobservable shift of the hypercharge gauge coupling, while the hidden $U(1)_{X}$ gauge boson and gaugino completely decouple from the observable sector [29]. However, in presence of SUSY breaking effects, the decoupling of the gaugino is not complete any more [21]. In the component formalism, the relevant part of the Lagrangian, including the supersymmetry breaking soft masses, reads

$$
\begin{aligned}
& \mathcal{L}_{\text {gauge }}=-\frac{1}{4}\left(\begin{array}{ll}
\hat{X}_{\mu \nu} & \hat{B}_{\mu \nu}
\end{array}\right) \mathcal{K}\left(\begin{array}{c}
\hat{X}^{\mu \nu} \\
\hat{B}^{\mu \nu}
\end{array}\right)-i\left(\begin{array}{ll}
\hat{\lambda}_{X} & \hat{\lambda}_{B}
\end{array}\right) \mathcal{K} \sigma^{\mu} \partial_{\mu}\left(\begin{array}{c}
\hat{\lambda}_{X}^{\dagger} \\
\hat{\lambda}_{B}^{\dagger}
\end{array}\right)
\end{aligned}
$$

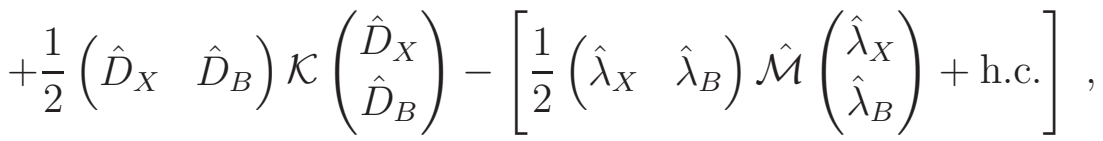

where $\mathcal{K}$ and $\hat{\mathcal{M}}$ denote, respectively, the kinetic and mass mixing matrices

$$
\mathcal{K}=\left(\begin{array}{cc}
1 & \chi \\
\chi & 1
\end{array}\right) \quad \text { and } \quad \hat{\mathcal{M}}=\left(\begin{array}{cc}
\hat{M}_{X} & \delta \hat{M} \\
\delta \hat{M} & \hat{M}_{B}
\end{array}\right)
$$

and $\hat{\lambda}_{X / B}$ and $\hat{D}_{X / B}$ are the gauginos and D-terms corresponding to the gauge fields $\hat{X}_{\mu \nu}$ and $\hat{B}_{\mu \nu}$, respectively. Note that the generation of mass mixing in general depends on details of the underlying theory.

In the basis where the kinetic terms are canonical, which can be achieved by the redefinition (which also holds for the corresponding $D$-terms and the vector bosons)

$$
\left(\begin{array}{l}
\hat{\lambda}_{X} \\
\hat{\lambda}_{B}
\end{array}\right)=\left(\begin{array}{cc}
1 & -\frac{\chi}{\sqrt{1-\chi^{2}}} \\
0 & \frac{1}{\sqrt{1-\chi^{2}}}
\end{array}\right)\left(\begin{array}{l}
\tilde{\lambda}_{X} \\
\tilde{\lambda}_{B}
\end{array}\right)
$$

the hidden gaugino and the four MSSM neutralinos mix, and the corresponding extended 
$(5 \times 5)$ neutralino mass matrix reads, to lowest order in $\chi$,

$$
\mathcal{M}_{\mathrm{N}}=\left(\begin{array}{ccccc}
M_{X} & \delta M & 0 & 0 & 0 \\
\delta M & M_{B} & 0 & -M_{Z} c_{\beta} s_{W} & M_{Z} s_{\beta} s_{W} \\
0 & 0 & M_{W} & M_{Z} c_{\beta} c_{W} & -M_{Z} s_{\beta} c_{W} \\
0 & -M_{Z} c_{\beta} s_{W} & M_{Z} c_{\beta} c_{W} & 0 & -\mu \\
0 & M_{Z} s_{\beta} s_{W} & -M_{Z} s_{\beta} c_{W} & -\mu & 0
\end{array}\right),
$$

where $\delta M \simeq \delta \hat{M}-\chi \hat{M}_{X}, M_{X} \simeq \hat{M}_{X}$ and $M_{B} \simeq \hat{M}_{B}$. Here, $\mu$ denotes the MSSM $\mu$-term, $M_{Z}$ the mass of the $Z^{0}$ gauge boson, $s_{W}$ the sine of the Weinberg angle and $s_{\beta}$ is related to the ratio of the two Higgs VEVs.

Upon diagonalization of the previous mass matrix we find a mass eigenstate $X=$ $\tilde{\lambda}_{X}+\Theta \tilde{\lambda}_{B}$ with mass $m_{X} \simeq M_{X}$, namely it is mostly hidden fermion with a small bino admixture given by

$$
\Theta \simeq \frac{\delta M}{M_{B}-M_{X}},
$$

from which follows that typically $\Theta \sim \mathcal{O}(\chi)$. Thus, even though the canonically normalized fields $\tilde{X}_{\mu \nu}$ and $\tilde{D}_{X}$ completely decouple from the observable sector, the mass eigenstate $X$, couples to the observable sector (e.g. the right-handed stau $\tilde{\tau}_{R}$ ) through the tiny bino component

$$
-\mathcal{L} \supset \sqrt{2} g^{\prime} Y_{\tilde{\tau}_{R}}\left(\overline{\tilde{\lambda}}_{B} P_{R} \tau\right) \widetilde{\tau}_{R} \rightarrow \sqrt{2} g^{\prime} Y_{\tilde{\tau}_{R}} \Theta\left(\bar{X} P_{R} \tau\right) \widetilde{\tau}_{R} .
$$

The presence of an additional $U(1)_{X}$ gauge group opens the stau decay channel $\widetilde{\tau}_{1} \rightarrow \tau X$ (we assume that $\widetilde{\tau}_{1} \simeq \tilde{\tau}_{R}$ ), with lifetime

$$
\Gamma_{\widetilde{\tau}_{1} \rightarrow \tau X}=\frac{g^{\prime 2}}{8 \pi} \Theta^{2} Y_{\tilde{\tau}_{R}}^{2} m_{\widetilde{\tau}_{1}}\left(1-\frac{m_{X}^{2}}{m_{\widetilde{\tau}_{1}}^{2}}\right)^{2} .
$$

In contrast to the scenario discussed in Sec. 2, where only the minimal effects of the coupling (2.1) where taken into account, many additional channels for thermal production are now open, since the $U(1)_{X}$ inherits all couplings of the hypercharge bino. The thermal production of hidden gauginos from $2 \rightarrow 2$ scattering processes was calculated in Ref. [21] and yielded an upper bound on the mixing angle $\Theta$ that is given by (provided that $X$ is stable)

$$
\Theta \lesssim 3 \times 10^{-12} \sqrt{\frac{m_{\tilde{q}}}{m_{X}}}
$$

where $m_{\tilde{q}}$ denotes the squark masses. In this calculation the dominant scattering processes involving one QCD and one hypercharge vertex were taken into account. Additional contributions from $1 \rightarrow 2$ processes were discussed in Ref. [20], yielding a relic 
density according to Eq. (2.9), when identifying $\lambda_{\widetilde{\tau}_{1}}^{2}=g^{\prime 2} Y_{\widetilde{\tau}_{R}}^{2} \Theta^{2}$ and

$$
\delta=\sum_{\tilde{f} \neq \widetilde{\tau}_{1}} \frac{Y_{\tilde{f}}^{2}}{Y_{\tilde{\tau}_{R}}^{2}} \frac{m_{\widetilde{\tau}_{1}}}{m_{\tilde{f}}}\left(\frac{1-m_{X}^{2} / m_{\tilde{f}}^{2}}{1-m_{X}^{2} / m_{\tilde{\tau}_{1}}^{2}}\right)^{2}
$$

where $\tilde{f}$ runs over all sfermions of the MSSM. For a typical SUSY mass spectrum, one obtains $\delta \sim \mathcal{O}(3-10)$. For stable $X$, this yields an upper bound of

$$
\Theta \lesssim 10^{-12} \sqrt{\frac{m_{\widetilde{\tau}_{1}}}{m_{X}}}
$$

Note that both contributions from $1 \rightarrow 2$ and $2 \rightarrow 2$ processes are roughly of the same order of magnitude and not much stronger than the production coming only from a term like Eq. (2.1) alone.

Bounds on the mixing parameter $\Theta$ are illustrated in Fig. 5. From there it is clear that a kinetically mixed hidden $U(1)_{X}$ gauge group with mixing parameters $10^{-13} \lesssim \Theta \lesssim$ $10^{-10}$ satisfies all constraints. Apart from the somewhat stronger thermal production, the situation is similar to what is shown in Fig. 2 and 3 .

The required mixing parameter $\Theta \sim \chi$ lies in the broad range of values that can be accommodated in string motivated $U(1)_{X}$ extensions: Without additional symmetries, the kinetic mixing $\chi$ is generically generated on one-loop level by integrating out chiral superfields charged under both, visible and hidden sector. In this case it acquires values typically around $\chi \sim 10^{-4}-10^{-2}$, corresponding to one-loop suppression [29, 30]. However, in compactifications of heterotic and type II strings, much smaller mixings are

possible [31, 32, 33, 34, 35]. For example, a lower bound around $\chi \gtrsim 10^{-16}$ was argued to hold in cases of gauge mediated supersymmetry breaking in heterotic string models [31, whereas in type-II models with warped extra dimensions the kinetic mixing parameter could be parametrically even smaller [32. Hence, scenarios with hidden unbroken $U(1)_{X}$ gauge groups provide simple and natural scenarios where the tension between thermal leptogenesis and gravitino dark matter is solved [21].

\subsection{A model for small couplings from non-renormalizable op- erators}

Let us now consider the case where the hidden fermion $X$ is part of a chiral supermultiplet, singlet under the Standard Model gauge group. Then, the gauge symmetry allows the superpotential term

$$
W \supset \frac{\alpha}{M} L H_{d} e_{R}^{c} X
$$



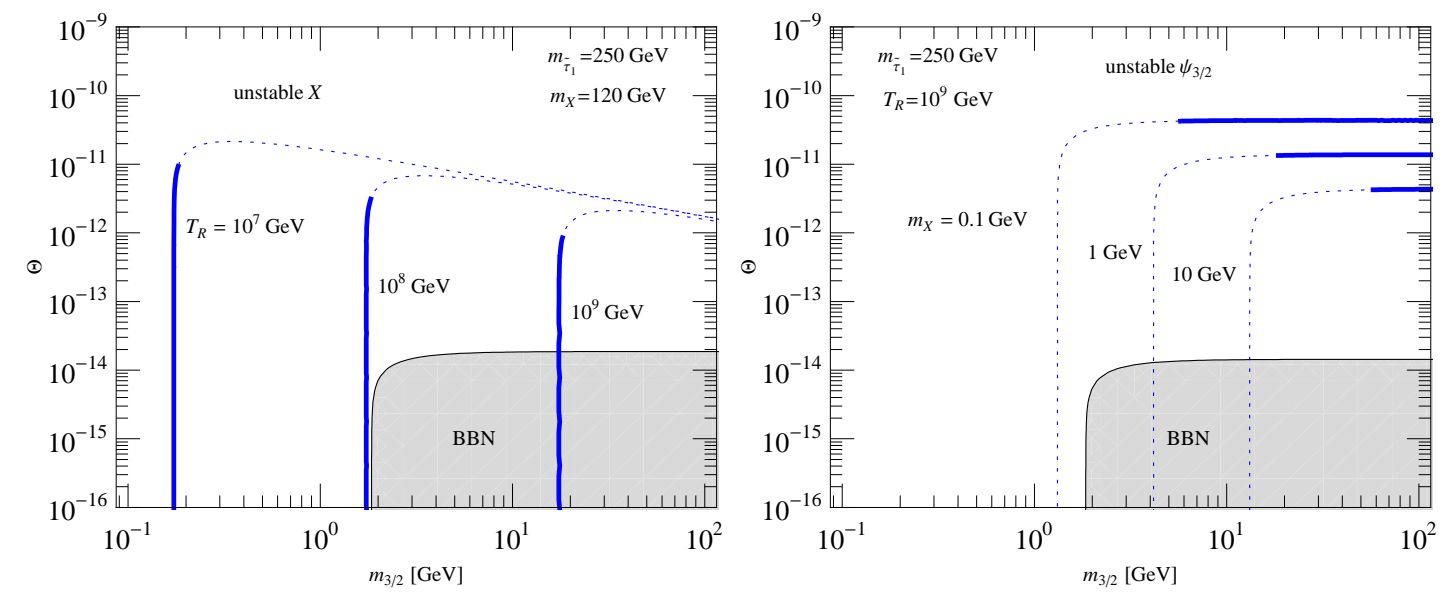

Figure 5: Constraints on the parameter space of the hidden $U(1)_{X}$ gaugino scenario with a $\widetilde{\tau}_{1}$ LOSP, for the case of gravitino LSPs (left panel, cf. Fig. 2) and for hidden gaugino LSPs (right panel, cf. Fig. 3), as function of the mixing parameter $\Theta$ and the gravitino mass $m_{3 / 2}$, and for $\widetilde{\tau}_{1}$ mass fixed as indicated. In the left panel the reheating temperature varies and whereas in the right panel the hidden gaugino mass $m_{X}$ varies. In both cases the NLSP is unstable and decays into the LSP, since the hidden vector boson remains massless.

This term leads, after the electroweak symmetry breaking, to a Lagrangian term of the form Eq. (2.1), with a Yukawa coupling which reads

$$
\lambda_{\widetilde{\tau}_{1}} \sim \frac{\alpha_{33}\left\langle H_{d}\right\rangle}{M} \sim 10^{-14} \alpha_{33} \cos \beta\left(\frac{M}{10^{16} \mathrm{GeV}}\right)^{-1} .
$$

It is remarkable that this value for the coupling lies in the allowed region of Figs. 2 and 3 if the non-renormalizable operator is suppressed by masses close to the Grand Unification scale.

The superpotential Eq.(3.11) can naturally arise from the decoupling of heavy particles with a mass of $\mathcal{O}(M)$. Let us consider an extension of the MSSM with three additional chiral superfields: $X(1,1,0), H_{u}^{\prime}(1,2,1)$ and $H_{d}^{\prime}(1,2,-1)$, where in parenthesis we indicate the quantum numbers under $S U(3)_{c} \times S U(2)_{L} \times U(1)_{Y}$. Note that $H_{u}^{\prime}$ and $H_{d}^{\prime}$ have identical gauge quantum numbers as the MSSM Higgs doublets $H_{u}$ and $H_{d}$, respectively. To avoid unwanted terms, we will further impose the following Peccei-Quinn 
transformation on the superfields:

$$
\begin{aligned}
\left(Q, U_{R}^{c}, D_{R}^{c}, L, E_{R}^{c}, N_{R}^{c}\right) & \rightarrow e^{i \alpha}\left(Q, U_{R}^{c}, D_{R}^{c}, L, E_{R}^{c}, N_{R}^{c}\right), \\
\left(H_{u}, H_{d}\right) & \rightarrow e^{-2 i \alpha}\left(H_{u}, H_{d}\right), \\
H_{u}^{\prime} & \rightarrow e^{2 i \alpha} H_{u}^{\prime} \\
H_{d}^{\prime} & \rightarrow e^{-2 i \alpha} H_{d}^{\prime} \\
X & \rightarrow X .
\end{aligned}
$$

This symmetry forbids the bilinear term $H_{u} H_{d}$ in the superpotential as well as a Majorana mass term for the right-handed neutrinos. The bilinear $\mu$ term could be generated via the Giudice-Masiero mechanism [36]. On the other hand, in order to generate the right-handed neutrino masses, we will further introduce a Standard Model singlet, $\Phi$, which transforms under the Peccei-Quinn symmetry as $\Phi \rightarrow e^{-2 i \alpha} \Phi$. Then, the term $\Phi N_{R}^{c} N_{R}^{c}$ is allowed in the superpotential and leads to right-handed Majorana masses if the scalar component of $\Phi$ acquires a vacuum expectation value, $\langle\Phi\rangle \sim M_{R}$. Note that even with the presence of the new field it is not possible to generate a bilinear term $H_{u} H_{d}$ in the superpotential 6

Then, the renormalizable superpotential of the model reads

$$
\begin{aligned}
W= & L\left(\lambda_{E} \cdot H_{D}\right) E_{R}^{c}+Q\left(\lambda_{D} \cdot H_{D}\right) D_{R}^{c}+\lambda_{u} Q H_{u} U_{R}^{c}+\lambda_{\nu} L H_{u} N_{R}^{c}+\lambda_{\mathrm{M}} \Phi N_{R}^{c} N_{R}^{c} \\
& +\left(\mu_{D} \cdot H_{D}\right) H_{u}^{\prime}+\left(\lambda_{X} \cdot H_{D}\right) H_{u}^{\prime} X+M_{X} X X+\kappa_{X} X^{3}+C_{X} X+\ldots,
\end{aligned}
$$

where the ellipsis indicates additional terms in the superpotential which lead to the breaking of supersymmetry and the breaking of the Peccei-Quinn symmetry. Here, we used a compact notation where $H_{D}=\left(H_{d}, H_{d}^{\prime}\right), \lambda_{E} \cdot H_{D}=\lambda_{e} H_{d}+\lambda_{e}^{\prime} H_{d}^{\prime}, \lambda_{X} \cdot H_{D}=\lambda_{x} H_{d}+$ $\lambda_{x}^{\prime} H_{d}^{\prime}$ etc. The linear term in $X$ can be eliminated by a shift, $X \rightarrow X-C_{X} /\left(2 M_{X}\right)$. Upon redefining the parameters, this amounts to setting $C_{X}=0$. In general $\mu_{D i} \sim \mathcal{O}\left(M_{\mathrm{GUT}}\right)$, although we will choose to work in the basis in the $\left(H_{d}, H_{d}^{\prime}\right)$-space such that $\mu_{D}=(0, M)$, being $M \sim \mathcal{O}\left(M_{\mathrm{GUT}}\right)$. Written in components, we thus have

$$
\begin{aligned}
W= & W_{\mathrm{MSSM}}^{\text {Yukawa }}+\lambda_{\nu} L H_{u} N_{R}^{c}+\lambda_{\mathrm{M}} \Phi N_{R}^{c} N_{R}^{c} \\
& +\lambda_{e}^{\prime} L H_{d}^{\prime} E_{R}^{c}+\lambda_{d}^{\prime} Q H_{d}^{\prime} D_{R}^{c}+M H_{d}^{\prime} H_{u}^{\prime}+\left(\lambda_{x} H_{d}+\lambda_{x}^{\prime} H_{d}^{\prime}\right) H_{u}^{\prime} X \\
& +M_{X} X X+\kappa_{X} X^{3}+\ldots
\end{aligned}
$$

\footnotetext{
${ }^{6}$ In the presence of the field $\Phi$ new, non-renormalizable, terms appear in the superpotential suppressed by a large mass scale, $M_{*}$, such as $Q Q Q L \Phi^{2} / M_{*}^{3}$. After the breaking of the Peccei-Quinn symmetry this term leads to a superpotential term $\left(M_{R} / M_{*}\right)^{2} Q Q Q L / M_{*}$ which induces proton decay. However, the small factor $\left(M_{R} / M_{*}\right)^{2}$ and the plausibly small coefficients of the dimension-7 operator for the first generation can yield a proton lifetime in agreement with the stringent experimental bounds.
} 
The breaking of the Peccei-Quinn symmetry at intermediate scales leads to Majorana masses for the right-handed neutrinos, $M_{\mathrm{M}}$. Then, the decoupling of the heavy fields $H_{u}^{\prime}$, $H_{d}^{\prime}, N_{R}^{c}$ finally leads to the following effective superpotential

$$
\begin{aligned}
W_{\mathrm{eff}}= & W_{\mathrm{MSSM}}^{\text {Yukawa }}-\left(\lambda_{\nu} M_{M}^{-1} \lambda_{\nu}^{T}\right)\left(L H_{d}\right)\left(L H_{d}\right) \\
& -\frac{\lambda_{e}^{\prime} \lambda_{x}}{M} L H_{d} E_{R}^{c} X-\frac{\lambda_{d}^{\prime} \lambda_{x}}{M} Q H_{d} D_{R}^{c} X+M_{X} X X+\kappa_{X} X^{3}
\end{aligned}
$$

which contains the term Eq. (3.11). Note that this model is free from gauge anomalies and preserves the successful MSSM gauge coupling unification.

In this model, there are additional contributions to the thermal production of the hidden fermion $X$ from $2 \rightarrow 2$ scattering processes, such as $\widetilde{\tau} h^{0} \rightarrow X \tau$ and $\widetilde{\tau} \rightarrow X h^{0}$. These processes stem from the higher-dimensional operators (HDO) in Eq. (3.20), and the amplitude for these processes increases with energy squared, $|\mathcal{M}|_{H D O}^{2} \sim s / M^{2}$, where $s$ denotes the square of the center-of-mass energy. Therefore these production channels can be very efficient at the high temperatures required by thermal leptogenesis. We will now shortly discuss the impact of these production channels on the overproduction constraints.

The sum of the matrix elements for all possible scatterings producing an $X$ or an $\tilde{X}$, respectively, are

$$
\sum_{\text {channels }}|\mathcal{M}|_{12 \rightarrow 3 X}^{2}=\sum_{\text {channels }}|\mathcal{M}|_{12 \rightarrow 3 \tilde{X}}^{2}=12 s \times \frac{\lambda_{x}^{2}}{M^{2}} \sum_{i, j}\left(\left(\lambda_{e}^{\prime}\right)_{i j}^{2}+3\left(\lambda_{d}^{\prime}\right)_{i j}^{2}\right),
$$

where we also summed over the initial and final-state spins. The effective $X$-stau-tau coupling can be identified with

$$
\lambda_{\widetilde{\tau}_{1}}^{2} \equiv \frac{\lambda_{x}^{2} v_{d}^{2}}{M^{2}} \sum_{i}\left(\lambda_{e}^{\prime}\right)_{i \tau}^{2} \equiv \frac{\alpha^{2} v_{d}^{2}}{M^{2}}
$$

where $v_{d}=v \cdot \cos \beta \simeq \cos \beta 175 \mathrm{GeV}$. Assuming for simplicity that all other Yukawa couplings are zero (which will give the minimal contribution to the UV thermal production), the hidden fermion yield reads [20]

$$
\begin{aligned}
Y_{H D O} & \simeq 3 \times 12 \times \frac{0.4 T_{R} \alpha^{2} \sqrt{8 \pi} M_{P}}{\pi^{7} M^{2} g_{*}^{3 / 2}} \\
& =1.7 \times 10^{-10} \alpha^{2}\left(\frac{T_{R}}{10^{9} \mathrm{GeV}}\right)\left(\frac{10^{16} \mathrm{GeV}}{M}\right)^{2}\left(\frac{915 / 4}{g_{*}}\right)^{3 / 2}
\end{aligned}
$$

where $M_{P} \simeq 2.4 \times 10^{18} \mathrm{GeV}$ denotes the reduced Planck mass. In this expression the prefactor 3 takes into account that the hidden fermions are produced in two ways: the scattering $12 \rightarrow 3 X$, which produces just one hidden fermion, and the scattering $12 \rightarrow$ 
$3 \tilde{X}$, which produces two hidden fermions, due to the fast decay $\tilde{X} \rightarrow X X$ (unless $\kappa_{X} \ll$ $10^{-12}$ ). Assuming that $X$ is stable on cosmological time-scales, this leads to an abundance

$$
\Omega_{X}^{H D O} h^{2} \simeq 0.045 \alpha^{2}\left(\frac{m_{X}}{1 \mathrm{GeV}}\right)\left(\frac{T_{R}}{10^{9} \mathrm{GeV}}\right)\left(\frac{10^{16} \mathrm{GeV}}{M}\right)^{2}\left(\frac{915 / 4}{g_{*}}\right)^{3 / 2} .
$$

For comparison with the freeze-in abundance (2.9), it is instructive to express this in terms of the effective coupling parameter $\lambda_{\widetilde{\tau}_{1}}$. Then, with $g_{*}=915 / 4$,

$$
\Omega_{X}^{H D O} h^{2} \simeq 0.11 \cos ^{-2} \beta\left(\frac{m_{X}}{1 \mathrm{GeV}}\right)\left(\frac{T_{R}}{10^{9} \mathrm{GeV}}\right)\left(\frac{\lambda_{\widetilde{\tau}_{1}}}{2.7 \times 10^{-14}}\right)^{2} .
$$

From requiring $\Omega_{X}^{H D O} h^{2}<0.11$ one thus obtains a bound

$$
\left|\lambda_{\widetilde{\tau}_{1}}\right| \lesssim 2.7 \times 10^{-14} \cos \beta\left(\frac{1 \mathrm{GeV}}{m_{X}}\right)^{\frac{1}{2}}\left(\frac{10^{9} \mathrm{GeV}}{T_{R}}\right)^{\frac{1}{2}}
$$

which depends on the reheating temperature $T_{R}$ and the mass of the hidden fermion $m_{X}$.

Bounds on the above model, for the case where the hidden fermion mass is very small, are summarized in Fig. [ (note that gravitino LSPs together with reheating temperatures $T_{R} \sim 10^{9} \mathrm{GeV}$ are excluded in the present model due to overproduction of the hidden fermion NLSPs). We show results for unstable gravitinos only, since even a very small $\kappa_{X}$ makes the gravitino unstable at cosmological timescales, due to the fast decay $\psi_{3 / 2} \rightarrow$ $X X X$. When comparing Fig. 6] with Fig. 3, it is apparent that the contribution to the relic abundance of hidden fermions coming from the HDO, $c f$. Eq. (3.23), reduces the allowed parameter space for the coupling $\lambda_{\widetilde{\tau}_{1}}$ considerably. These ultraviolet contributions depend linearly on the reheating temperature and dominate the infrared contributions coming from the renormalizable operator. They can potentially reintroduce the gravitino problem, cf. Eq. (1.1), if the mass of the hidden fermion is too large. However, for small hidden fermion masses $m_{X} \lesssim 1 \mathrm{GeV}$ all constraints from overproduction, BBN and structure formation can be simultaneously satisfied for couplings in the range $\lambda_{\widetilde{\tau}_{1}} \sim$ $10^{-14}-10^{-13}$. Most interestingly, these couplings are preferred if the non-renormalizable operator is suppressed by masses close to the GUT scale, see Eq. (3.12).

\section{Experimental signatures}

In this section we will briefly discuss possible experimental signatures of the scenario proposed above at colliders and in cosmic-ray observations.

In this scenario the coupling constant of the LOSP to the hidden fermion $X$ is typically smaller than $10^{-12}$, therefore the decay length is much larger than the size of 


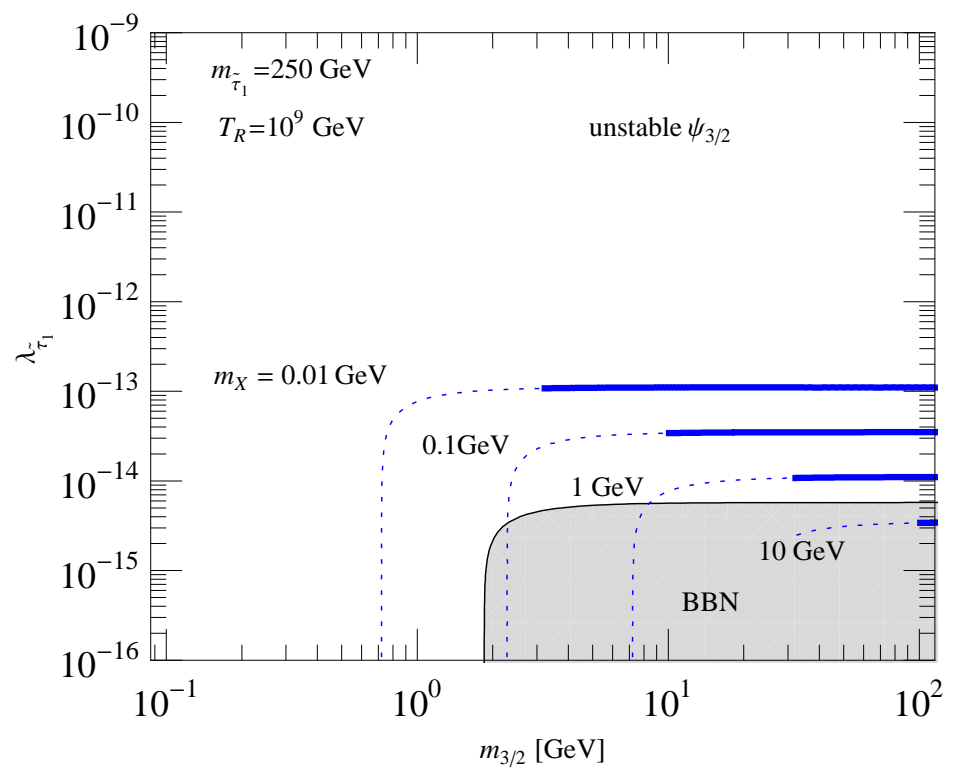

Figure 6: Similar to Fig. 3, but for the model described in Section. 3.2, taking into account also the minimal ultraviolet contributions to the $X$ production, and assuming $\tan \beta=2$. We show constraints for the case where the gravitino is the NLSP and decays into $X$ (in contrast to the previous figures the gravitino decays here into three hidden fermions, $\left.\psi_{3 / 2} \rightarrow X X X\right)$. The reheating temperature and the $\widetilde{\tau}_{1}$ mass are fixed to $10^{9} \mathrm{GeV}$ and $250 \mathrm{GeV}$, respectively, whereas the hidden fermion $X$ mass varies in the range $m_{X}=$ 0.01-10 GeV. Note that a typical value for $\lambda_{\widetilde{\tau}_{1}}$ in our model is $\sim 10^{-14}-10^{-13}$ when the non-renormalizable operator is suppressed by masses close to the GUT scale, $c f$. Eq.(3.12).

typical collider detectors. As a consequence, if the LOSP is the lightest neutralino, the experimental signatures at colliders are identical to the case of the MSSM with R-parity conserved, since the lightest neutralino just escapes the detector. On the other hand, if the LOSP is the lightest stau, it propagates through the detector leaving a heavily ionizing charged track [37]. Furthermore, if the stau velocity is small enough, it could get trapped in the detector and decay eventually, producing a tau moving in a non-radial direction. This signature, albeit very spectacular, is not specific of this scenario but also arises in scenarios with stau LOSP and gravitino or axino LSP [38].

A hint towards our proposed scenario arises from the measurement of the coupling stau-tau-hidden fermion. More concretely, at colliders it will be possible a determination of the stau mass and the stau lifetime, from which the coupling could be determined 
through Eq. (2.3):

$$
\left|\lambda_{\widetilde{\tau}_{1}}\right| \simeq \sqrt{\frac{8 \pi}{\tau_{\widetilde{\tau}_{1}} m_{\widetilde{\tau}_{1}}}}
$$

If the coupling inferred from collider experiments lies in the range preferred by cosmology, Eq. (2.11), and if the lifetime $\tau_{\widetilde{\tau}_{1}}$ is in agreement with BBN bounds, this scenario would gain strength.

Furthermore, if the small coupling of the stau to the tau and the hidden fermion is due to the non-renormalizable operator Eq.(3.11), then a very interesting signature could be observed at colliders: after being stopped in the detector, the stau could decay producing also a Higgs boson, with branching ratio:

$$
\operatorname{BR}\left(\widetilde{\tau}_{1} \rightarrow \tau h X\right) \simeq \frac{\tan ^{2} \beta \sin ^{2} \alpha}{384 \pi^{2}} \frac{m_{\widetilde{\tau}_{1}}^{2}}{v^{2}} f\left(m_{h}^{2} / m_{\widetilde{\tau}_{1}}^{2}\right)
$$

where $f(r)=1+9 r-9 r^{2}-r^{3}+6 r(1+r) \ln (r), v=175 \mathrm{GeV}$ is the Higgs vacuum expectation value and $\alpha$ is the angle that diagonalizes the CP-even Higgs squared-mass matrix. For typical values of the light Higgs mass and the stau mass, we obtain

$$
\operatorname{BR}\left(\widetilde{\tau}_{1} \rightarrow \tau h X\right) \simeq 0.006\left(\frac{m_{\widetilde{\tau}_{1}}}{250 \mathrm{GeV}}\right)^{2}\left(\frac{\tan \beta}{10}\right)^{2} \sin ^{2} \alpha,
$$

where we have taken $m_{h} / m_{\widetilde{\tau}_{1}}=115 / 250$ in the argument of the function $f$. Therefore, if 1000 staus could be stopped, a few Higgs events could be observed in the detector.

For comparison, in scenarios with gravitino LSP and stau NLSP, the Higgs decay is strongly suppressed by the tau Yukawa coupling. The branching ratio, taking Higgsstrahlung into account, reads

$$
\operatorname{BR}\left(\widetilde{\tau}_{1} \rightarrow \tau h \psi_{3 / 2}\right) \simeq \frac{\tan ^{2} \beta \sin ^{2} \alpha}{64 \pi^{2}} \frac{m_{\tau}^{2}}{v^{2}} g\left(m_{h}^{2} / m_{\widetilde{\tau}_{1}}^{2}\right),
$$

where $g(r)=\left(1+8 r+6 r^{2}\right) \ln \left(r^{-1}\right)-\left(43+80 r-108 r^{2}-16 r^{3}+r^{4}\right) / 12$, and we assumed $m_{3 / 2} \ll m_{\widetilde{\tau}_{1}}$. Again, inserting typical values of parameters,

$$
\mathrm{BR}\left(\widetilde{\tau}_{1} \rightarrow \tau h \psi_{3 / 2}\right) \simeq 3.4 \times 10^{-7}\left(\frac{\tan \beta}{10}\right)^{2} \sin ^{2} \alpha,
$$

which is suppressed by the tau mass, making the observation of Higgs events from stopped staus more difficult. Thus, the number of Higgs events in late stau decays constitutes a way to discriminate between a scenario with gravitino/axino LSP and a scenario where a hidden fermion couples to the stau and the tau via the non-renormalizable operator Eq. (3.11).

Another possible experimental signature in this scenario is the observation of a large number of lepton flavour violating stau-LOSP decays. In the model described in section 
3.2 it is in general not possible to simultaneously diagonalize the usual MSSM coupling $\lambda_{e} L H_{d} E_{R}^{c}$ and the new coupling with the heavy Higgs doublet $\lambda_{e}^{\prime} L H_{d}^{\prime} E_{R}^{c}$. As a consequence, the effective coupling in the Lagrangian $\left(\lambda_{e}^{\prime}\right)_{i j} \lambda_{x}\left\langle H_{d}\right\rangle / M \bar{L}_{i} \tilde{E}_{R j}^{c} X$ in general contains sizeable off-diagonal entries, which induce at tree level the lepton flavour violating decays $\widetilde{\tau}_{1} \rightarrow \mu X$ and $\widetilde{\tau}_{1} \rightarrow e X$ with branching ratio:

$$
\frac{\operatorname{BR}\left(\widetilde{\tau}_{1} \rightarrow \ell_{i} X\right)}{\operatorname{BR}\left(\widetilde{\tau}_{1} \rightarrow \tau X\right)}=\left|\frac{\left(\lambda_{e}^{\prime}\right)_{i 3}}{\left(\lambda_{e}^{\prime}\right)_{33}}\right|^{2} .
$$

Future colliders are capable of detecting the electrons and muons produced in the decay of long lived staus if $\left(\lambda_{e}^{\prime}\right)_{i 3} /\left(\lambda_{e}^{\prime}\right)_{33}$ is larger than $\sim 3 \times 10^{-2}\left(9 \times 10^{-3}\right)$ provided $3 \times 10^{3}\left(3 \times 10^{4}\right)$ staus can be collected [39]. Therefore, this scenario offers good prospects to detect flavour violation in stau decays at future colliders.

It is important to note that this scenario can be easily compatible with the present experimental constraints on lepton flavour violation in the charged lepton sector. Namely, the process $\mu \rightarrow e \gamma$ induced at the one loop level via the Yukawa coupling $\lambda_{e}^{\prime}$ is strongly suppressed due to the large mass of $H_{d}^{\prime}$. Furthermore, if the scale of mediation of SUSY breaking is larger than the mass of the heavy Higgs doublets, which is the case for $m_{3 / 2} \gtrsim 10 \mathrm{GeV}\left(M / 10^{16} \mathrm{GeV}\right)$, the couplings $\lambda_{e}^{\prime}$ and $\lambda_{d}^{\prime}$ induce off-diagonal entries in the squark and slepton mass matrices through renormalization group running, which in turn induce flavour violating processes in the quark and lepton sector through quantum effects. However, these effects appear then at the two loop level and are naturally suppressed. The most stringent bound coming from $\mu \rightarrow e \gamma$ implies mild constraints of the order $\left(\lambda_{e}^{\prime \dagger} \lambda_{e}^{\prime}\right)_{21} \lesssim 0.1\left(m_{\widetilde{\tau}_{1}} / 250 \mathrm{GeV}\right)^{2}$, whereas the 31 and 32 entries can be order one. Moreover, these effects can be further suppressed by appropriate choices of the flavour structure of the matrices $\lambda_{e}^{\prime}$ and $\lambda_{d}^{\prime}$. Therefore, in the scenario proposed in section 3.2 it is expected that the stopped staus will decay not only into taus, but also into muons and electrons with a large rate, while being consistent with all present constraints on lepton flavour violation. In contrast, in scenarios with gravitino LSP and stau NLSP the rates of lepton flavour violating stau decays are predicted to be small [39]. Therefore, the number of lepton flavor violating stau decays offers a sensitive probe to discriminate gravitino LSP scenarios from the hidden fermion scenario discussed in section 3.2 .

In section 2.1.1 we discussed the possibility that the gravitino and the hidden fermion could be stable at cosmological timescales. Nevertheless, the heavier dark matter particle will eventually decay into the lightest and a tau-antitau pair with a decay rate which is doubly suppressed by the Planck mass and by the small coupling between the stau and the hidden fermion. Namely, when the gravitino is lighter than the hidden fermion, the 
latter decays with a rate which reads

$$
\Gamma\left(X \rightarrow \tau^{+} \tau^{-} \psi_{3 / 2}\right)=\frac{\left|\lambda_{\widetilde{\tau}_{1}}\right|^{2}}{18432 \pi^{3}} \frac{m_{X}^{9}}{M_{P}^{2} m_{3 / 2}^{2} m_{\widetilde{\tau}_{1}}^{4}},
$$

where we have assumed $m_{3 / 2} \ll m_{X}$. On the other hand, when the hidden fermion is lighter than the gravitino, the decay rate approximately reads [14]

$$
\Gamma\left(\psi_{3 / 2} \rightarrow \tau^{+} \tau^{-} X\right) \simeq \frac{\left|\lambda_{\widetilde{1}_{1}}\right|^{2}}{92160 \pi^{3}} \frac{m_{3 / 2}^{7}}{M_{P}^{2} m_{\widetilde{\tau}_{1}}^{4}} .
$$

The electrons, positrons, gamma rays and neutrinos produced in the decay could be in principle detected in cosmic-ray observations as an excess over the expected astrophysical backgrounds. For dark matter particles with a mass $\sim 100 \mathrm{GeV}$, the decay products are observable if the lifetime is shorter than $\sim 10^{26} \mathrm{~s}$ [40]. However, in our scenarios, the heavier component of dark matter has a lifetime

$$
\begin{gathered}
\tau\left(X \rightarrow \tau^{+} \tau^{-} \psi_{3 / 2}\right) \sim 10^{35} \mathrm{~s}\left(\frac{\left|\lambda_{\tilde{\tau}_{1}}\right|}{10^{-12}}\right)^{-2}\left(\frac{m_{X}}{100 \mathrm{GeV}}\right)^{-9}\left(\frac{m_{3 / 2}}{10 \mathrm{GeV}}\right)^{2}\left(\frac{m_{\tilde{\tau}_{1}}}{250 \mathrm{GeV}}\right)^{4}, \\
\tau\left(\psi_{3 / 2} \rightarrow \tau^{+} \tau^{-} X\right) \sim 10^{38} \mathrm{~s}\left(\frac{\left|\lambda_{\tilde{\tau}_{1}}\right|}{10^{-12}}\right)^{-2}\left(\frac{m_{3 / 2}}{100 \mathrm{GeV}}\right)^{-7}\left(\frac{m_{\tilde{\tau}_{1}}}{250 \mathrm{GeV}}\right)^{4},
\end{gathered}
$$

which have been normalized to typical values of the masses and couplings which yield a thermal history of the Universe incorporating thermal leptogenesis and successful BBN (cf. Fig. 2). Therefore, the cosmic-ray fluxes typically lie many orders of magnitude below the background, being completely unobservable.

\section{Conclusions}

Cosmological scenarios where the observed matter-antimatter asymmetry is generated by the supersymmetric thermal leptogenesis mechanism generically fail to reproduce the observed abundances of primordial elements. In the minimal scenario, the LSP must be a gravitino heavier than $\sim 5 \mathrm{GeV}$. Therefore, if R-parity is conserved, the NLSP is very long lived, jeopardizing the successful predictions of the standard BBN scenario. To solve this conflict we have postulated the existence of a light hidden sector fermion which couples very weakly to the NLSP. If the coupling is large enough, the NLSP will decay dominantly into hidden sector fermions before the epoch of primordial nucleosynthesis, avoiding all the nucleosynthesis constraints altogether. We have analyzed the constraints on this coupling from dark matter overproduction and from structure formation and we have found a wide window of parameters where the cosmological history of the Universe can be consistent with baryogenesis through thermal leptogenesis and with BBN. We have 
presented two concrete models to illustrate the viability of the above mentioned scenario. Furthermore, we have discussed some experimental signatures at particle colliders which can provide evidence for our mechanism and distinguish it from other scenarios. Examples of these are the number of Higgs events and the lepton flavour violation in late stau decays. Finally, we demonstrated that the scenario is easily compatible with bounds from cosmic-ray observations.

\section{Acknowledgements}

AI and CW would like to thank the IPMU for kind hospitality during the completion of this work. ADS gratefully thanks the warm hospitality of the Physics Department at TUM where part of this work was done. The work of MG and AI was partially supported by the DFG cluster of excellence "Origin and Structure of the Universe." The work of ADS was supported in part by the INFN "Bruno Rossi" Fellowship, and in part by the U.S. Department of Energy (DoE) under contract No. DE-FG02-05ER41360.

\section{Note added}

The authors of Ref. [41, which appeared simultaneously to this one, independently considered a similar mechanism to accommodate BBN bounds and thermal leptogenesis for a specific situation where a stau LOSP can decay to a goldstino.

\section{References}

[1] P. Minkowski, Phys. Lett. B 67 (1977) 421; M. Gell-Mann, P. Ramond and R. Slansky, Proceedings of the Supergravity Stony Brook Workshop, New York 1979, eds. P. Van Nieuwenhuizen and D. Freedman; T. Yanagida, Proceedinds of the Workshop on Unified Theories and Baryon Number in the Universe, Tsukuba, Japan 1979, eds. A. Sawada and A. Sugamoto; R. N. Mohapatra, G. Senjanovic, Phys.Rev.Lett. 44 (1980)912, ibid. Phys.Rev. D23 (1981) 165; S. L. Glashow, The Future Of Elementary Particle Physics, In *Cargese 1979, Proceedings, Quarks and Leptons*, 687-713 and Harvard Univ.Cambridge - HUTP-79-A059 (79,REC.DEC.) 40p; J. Schechter and J. W. F. Valle, Phys. Rev. D 22 (1980) 2227.

[2] M. Fukugita and T. Yanagida, Phys. Lett. B 174 (1986) 45. 
[3] S. Davidson and A. Ibarra, Phys. Lett. B 535 (2002) 25; W. Buchmuller, P. Di Bari and M. Plumacher, Annals Phys. 315 (2005) 305.

[4] M. Bolz, A. Brandenburg and W. Buchmüller, Nucl. Phys. B 606 (2001) 518 [Erratum-ibid. B 790 (2008) 336]; J. Pradler and F. D. Steffen, Phys. Rev. D 75, 023509 (2007); V. S. Rychkov and A. Strumia, Phys. Rev. D 75, 075011 (2007).

[5] M. Kawasaki, K. Kohri and T. Moroi, Phys. Rev. D 71 (2005) 083502.

[6] R. H. Cyburt, J. Ellis, B. D. Fields, F. Luo, K. A. Olive and V. C. Spanos, JCAP 0910 (2009) 021.

[7] E. Komatsu et al., arXiv:1001.4538 [astro-ph.CO].

[8] K. Jedamzik, Phys. Rev. D 74 (2006) 103509.

[9] M. Pospelov, Phys. Rev. Lett. 98 (2007) 231301.

[10] K. Kohri and F. Takayama, Phys. Rev. D 76, 063507 (2007); M. Kaplinghat and A. Rajaraman, Phys. Rev. D 74, 103004 (2006); R. H. Cyburt et al. JCAP 0611, 014 (2006); K. Hamaguchi et al. Phys. Lett. B 650 (2007) 268; J. Pradler and F. D. Steffen, Phys. Lett. B 666, 181 (2008); M. Pospelov, J. Pradler and F. D. Steffen, JCAP 0811 (2008) 020.

[11] K. Jedamzik and M. Pospelov, New J. Phys. 11 (2009) 105028.

[12] T. Kanzaki et al. Phys. Rev. D 75 (2007) 025011.

[13] J. L. Diaz-Cruz, J. R. Ellis, K. A. Olive and Y. Santoso, JHEP 0705 (2007) 003.

[14] W. Buchmüller, L. Covi, K. Hamaguchi, A. Ibarra and T. Yanagida, JHEP 0703, 037 (2007).

[15] M. Ratz, K. Schmidt-Hoberg and M. W. Winkler, JCAP 0810 (2008) 026.

[16] L. Boubekeur, K. Y. Choi, R. R. de Austri and O. Vives, JCAP 1004 (2010) 005;

J. Pradler and F. D. Steffen, Nucl. Phys. B 809 (2009) 318.

[17] J. Pradler and F. D. Steffen, Phys. Lett. B 648 (2007) 224.

[18] T. Asaka and T. Yanagida, Phys. Lett. B 494 (2000) 297.

[19] T. Asaka, K. Hamaguchi and K. Suzuki, Phys. Lett. B 490, 136 (2000).

[20] L. J. Hall, K. Jedamzik, J. March-Russell and S. M. West, JHEP 1003 (2010) 080. 
[21] A. Ibarra, A. Ringwald and C. Weniger, JCAP 0901 (2009) 003.

[22] E. W. Kolb and M. S. Turner, Front. Phys. 69 (1990) 1.

[23] P. McDonald et al. [SDSS Collaboration], Astrophys. J. Suppl. 163 (2006) 80.

[24] L. E. Strigari, M. Kaplinghat and J. S. Bullock, Phys. Rev. D 75 (2007) 061303;

F. Borzumati, T. Bringmann and P. Ullio, Phys. Rev. D 77 (2008) 063514.

[25] A. Boyarsky, J. Lesgourgues, O. Ruchayskiy and M. Viel, JCAP 0905 (2009) 012.

[26] M. Viel, M. G. Haehnelt and V. Springel, Mon. Not. Roy. Astron. Soc. 354, 684 (2004).

[27] J. L. Feng, S. Su and F. Takayama, Phys. Rev. D 70, 075019 (2004).

[28] N. Arkani-Hamed, A. Delgado and G. F. Giudice, Nucl. Phys. B 741 (2006) 108.

[29] B. Holdom, Phys. Lett. B 166, 196 (1986); B. Holdom, Phys. Lett. B 259 (1991) 329.

[30] F. del Aguila, G. D. Coughlan and M. Quiros, Nucl. Phys. B 307, 633 (1988) [Erratum-ibid. B 312, 751 (1989)].

[31] K. R. Dienes, C. F. Kolda and J. March-Russell, Nucl. Phys. B 492, 104 (1997).

[32] S. A. Abel, M. D. Goodsell, J. Jaeckel, V. V. Khoze and A. Ringwald, JHEP 0807, 124 (2008).

[33] M. Goodsell, J. Jaeckel, J. Redondo and A. Ringwald, JHEP 0911 (2009) 027.

[34] A. Arvanitaki, N. Craig, S. Dimopoulos, S. Dubovsky and J. March-Russell, arXiv:0909.5440 [hep-ph].

[35] M. Goodsell and A. Ringwald, arXiv:1002.1840 [hep-th].

[36] G. F. Giudice and A. Masiero, Phys. Lett. B 206 (1988) 480.

[37] M. Drees and X. Tata, Phys. Lett. B 252 (1990) 695.

[38] W. Buchmuller, K. Hamaguchi, M. Ratz and T. Yanagida, Phys. Lett. B 588 (2004) 90; J. L. Feng and B. T. Smith, Phys. Rev. D 71 (2005) 015004 [Erratum-ibid. D 71 (2005) 019904]; K. Hamaguchi, Y. Kuno, T. Nakaya and M. M. Nojiri, Phys. Rev. D 70 (2004) 115007; A. Brandenburg, L. Covi, K. Hamaguchi, L. Roszkowski and F. D. Steffen, Phys. Lett. B 617, 99 (2005). 
[39] K. Hamaguchi and A. Ibarra, JHEP 0502 (2005) 028 arXiv:hep-ph/0412229.

[40] A. Ibarra and D. Tran, JCAP 0902 (2009) 021; A. Ibarra, D. Tran and C. Weniger, JCAP 1001 (2010) 009, Phys. Rev. D 81 (2010) 023529.

[41] C. Cheung, J. Mardon, Y. Nomura and J. Thaler, arXiv:1004.4637 [hep-ph]. 